\section{Drs. Aggarwal and Oddis reply}

\section{To the Editor:}

We appreciate the thoughtful comments provided by Drs. Chen and Liu ${ }^{1}$, and Drs. Jearn and $\mathrm{Kim}^{2}$ in their respective letters in response to our manuscript ${ }^{3}$. They indeed provide helpful information and perspective in the interpretation of antinuclear antibody (ANA) testing and the reporting of cytoplasmic staining in laboratories outside of the United States. In principle, we all agree that it is helpful to recognize cytoplasmic staining patterns seen on HEp-2 ANA testing. Chen and Liu raise an important question about the definition of anticytoplasmic autoantibody (anti-CytAb) used in our study ${ }^{1}$. Our study simply focused on the potential recognition of antisynthetase antibody (anti-SynAb)-positive patients using the results of the screening ANA on HEp-2 cells. Our definition of positive anti-CytAb is cytoplasmic staining related to Jo1 and non-Jo1 antisynthetase autoantibodies, which includes the cytoplasmic dense fine speckled pattern, the cytoplasmic fine speckled pattern, and the cytoplasmic diffuse pattern ${ }^{4,5}$. We recognize that many sophisticated immunology laboratories can differentiate specific cytoplasmic patterns. However, many US immunology laboratories do not have the training or expertise to differentiate between various cytoplasmic patterns. We recommend the reporting of cytoplasmic staining simply as a screening test for the patient for whom the antisynthetase syndrome is being considered in the differential diagnosis. We further emphasize the importance of testing to confirm the presence of the specific antisynthetase autoantibody by immunoprecipitation or ELISA-based specific assays. Importantly, we believe that immunology laboratories should refrain from simply reporting a negative ANA, but should alert the ordering physician that cytoplasmic staining was identified if this pattern was seen by the immunology laboratory technician.

We agree with Jearn and Kim on the reporting of cytoplasmic staining for initial screening of anti-SynAb+ patients ${ }^{2}$. Although we agree with Jearn and Kim that various specific cytoplasmic staining patterns should be reported, we are not convinced that immunology laboratories around the world have the necessary training to report these patterns. We appreciate that Jearn and Kim clarify that anti-Ro can lead to cytoplasmic staining, which may explain a lower specificity for the antisynthetase syndrome. Moreover, given that anti-Ro is associated with various systemic autoimmune rheumatic disorders including systemic sclerosis, Sjögren syndrome, systemic lupus erythematosus, and myositis, it may indeed lead to false-positive cytoplasmic staining. Similarly, other cytoplasmic staining patterns may cause confusion in identifying the patient with the antisynthetase syndrome. Again, we emphasize that cytoplasmic staining should be used as a screening test only of the patient for whom the antisynthetase syndrome is being considered, but further confirmatory testing is necessary. Immunology laboratories should train their technicians to recognize different cytoplasmic patterns, perhaps providing more specific reporting of cytoplasmic-speckled patterns associated with the antisynthetase syndrome. Jearn and Kim claim that they have been partially able to overcome this problem by using a different substrate for ANA - human macrophage cell line, IT- $1^{6,7,8}$. However, most US immunology laboratories continue to use HEp-2 ANA for various reasons; therefore, we believe cytoplasmic pattern recognition on HEp-2 ANA test is still the most relevant to our population.

As noted by Jearn and Kim, nomenclature has been proposed for the prevention of misreporting and misinterpretation of the ANA. However, none of these proposed terms have been consistently used by immunology laboratories in the United States. We are encouraged to see efforts by the Korean Society for Laboratory Medicine to standardize the reporting of cytoplasmic patterns on routine ANA testing. Table 1 of Jearn and $\mathrm{Kim}^{2}$ shows that pattern recognition of even a single autoantibody (i.e., anti-Jo1, the most common and best-recognized antisynthetase autoantibody) remains challenging even after the quality improvement efforts of the Korean Society for Laboratory Medicine. It is also relevant that HEp-2 ANA kits by different companies may have discrepancies in titer and pattern because of differences in the substrates rather than variation by the reader. We look forward to formal publications on this topic.

ROHIT AGGARWAL, MD, MS, Division of Rheumatology and Clinical Immunology, University of Pittsburgh School of Medicine; CHESTER V. ODDIS, MD, Division of Rheumatology and Clinical Immunology, University of Pittsburgh School of Medicine, Pittsburgh, Pennsylvania, USA. Address correspondence to Dr. R. Aggarwal, UPMC Arthritis and Autoimmunity Center, Division of Rheumatology and Clinical Immunology, Department of Medicine, 3601 Fifth Ave., Suite 2B, Pittsburgh, Pennsylvania 15213, USA. E-mail: aggarwalr@upmc.edu, docrota@gmail.com

\section{REFERENCES}

1. Chen Y, Lui J. What is the definition of the anticytoplasmic autoantibody? J Rheumatol 2017;44:1564.

2. Jearn LH, Kim TY. Anti-tRNA synthetase-specific immunofluorescence patterns are easily detected in the suitable substrate. J Rheumatol 2017;44:1564-5.

3. Aggarwal R, Dhillon N, Fertig N, Koontz D, Qi Z, Oddis CV. A negative antinuclear antibody does not indicate autoantibody negativity in myositis: role of anticytoplasmic antibody as a screening test for antisynthetase syndrome. J Rheumatol 2017;44:223-9.

4. Chan EK, Damoiseaux J, Carballo OG, Conrad K, de Melo Cruvinel W, Francescantonio PL, et al. Report of the first international consensus on standardized nomenclature of antinuclear antibody HEp-2 cell patterns 2014-2015. Front Immunol 2015;6:412.

5. Damoiseaux J, von Mühlen CA, Garcia-De La Torre I, Carballo OG, de Melo Cruvinel W, Francescantonio PL, et al. International consensus on ANA patterns (ICAP): the bumpy road towards a consensus on reporting ANA results. Auto Immun Highlights 2016;7:1.

6. Jearn LH, Kim TY. AIT test has no problem in the detection of anti-ribosomal P. Arthritis Res Ther 2009;11:407.

7. Jearn LH, Kim TY. Anti-Jo1 antibody in polymyositis/ dermatomyositis is still closely associated with lung rather than joints. J Rheumatol 2015;42:1060.

8. Jearn LH, Kim TY. Should the external quality assessment sample of antinuclear antibodies be of a typical monospecific pattern? Clin Chem Lab Med 2010;48:1189-90.

J Rheumatol 2017;44:10; doi:10.3899/jrheum.170480 